\title{
Sensitivity of risk measures with respect to the normal approximation of total claim distributions
}

\section{Volker Krätschmer*} Henryk Zähle**

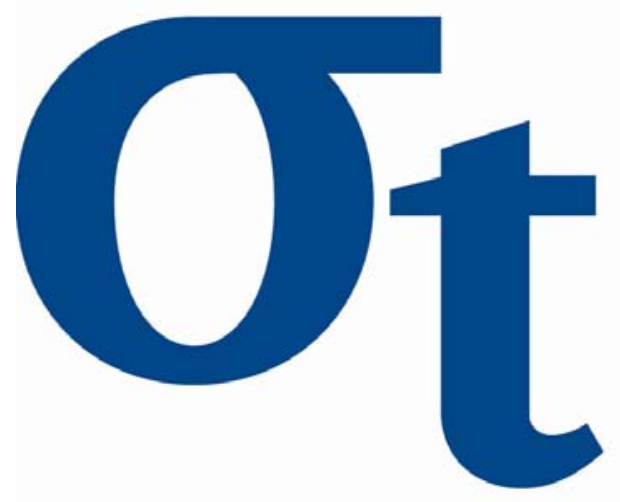

* Weierstrass Institute of Applied Analysis and Stochastics, Berlin, Germany

** Universität des Saarlandes, Saarbrücken, Germany

This research was supported by the Deutsche Forschungsgemeinschaft through the SFB 649 "Economic Risk". 


\title{
Sensitivity of risk measures with respect to the normal approximation of total claim distributions
}

\author{
Volker Krätschmer $^{a *}$ Henryk Zähle ${ }^{b}$ \\ ${ }^{a}$ Weierstrass Institute of Applied Analysis and Stochastics, Mohrenstrasse 39, D-10117 Berlin, \\ Germany; kraetsch@wias-berlin.de \\ ${ }^{b}$ Universität des Saarlandes, Fachrichtung Mathematik, Postfach 151150, 66041 Saarbrücken, \\ Germany; henryk.zaehle@freenet.de
}

\begin{abstract}
A simple and commonly used method to approximate the total claim distribution of a (possible weakly dependent) insurance collective is the normal approximation. In this article, we investigate the error made when the normal approximation is plugged in a fairly general distribution-invariant risk measure. We focus on the rate of the convergence of the error relative to the number of clients, we specify the relative error's asymptotic distribution, and we illustrate our results by means of a numerical example. Regarding the risk measure, we take into account distortion risk measures as well as distribution-invariant coherent risk measures.
\end{abstract}

Keywords: Total claim distribution, $\phi$ - and $\alpha$-mixing sequences of random variables, normal approximation, nonuniform Berry-Esseen inequality, distortion risk measure, coherent risk measure, robust representation.

JEL Classification: G22 G32

AMS 2000 Subject Classification: 60F05 62F12 91B30

*This research was partly supported by Deutsche Forschungsgemeinschaft through SFB 649 "Economic Risk" 


\section{Introduction}

In insurance practice, a simple and fast way to approximate the distribution of the total claim amount $S_{n}:=\sum_{i=1}^{n} X_{i}$ of a homogeneous (possibly weakly dependent) insurance collective $\left\{X_{1}, \ldots, X_{n}\right\}$ is the normal approximation. According to the CLT we have that the distribution of $S_{n}$ is "close" (relatively to $n$ ) to the normal distribution with mean $\mu_{n}:=\mathbb{E}\left[S_{n}\right]=n \mathbb{E}\left[X_{1}\right]$ and variance $\sigma_{n}^{2}:=\operatorname{Var}\left[S_{n}\right]$, provided the latter is finite. Practitioners then often plug this normal distribution $\mathcal{N}_{\mu_{n}, \sigma_{n}^{2}}$ in some distribution-invariant risk measure $\rho$ to approximate the collective's premium $\rho\left(F_{n}\right)$ by $\rho\left(\Phi_{n}\right)$, where $F_{n}$ and $\Phi_{n}:=\Phi_{\mu_{n}, \sigma_{n}^{2}}$ refer to the distribution functions (df) of $S_{n}$ and $\mathcal{N}_{\mu_{n}, \sigma_{n}^{2}}$, respectively. Actually, the values of $\mu_{n}$ and $\sigma_{n}$ have to be estimated by some suitable estimators $\hat{\mu}_{n}=\hat{\mu}_{n, m_{n}}$ and $\hat{\sigma}_{n}=\hat{\sigma}_{n, m_{n}}$ (based on a sample of size $m_{n}$ ) and the resulting (random) df $\hat{\Phi}_{n}:=\Phi_{\hat{\mu}_{n}, \hat{\sigma}_{n}^{2}}$ is plugged in the risk measure to approximate the collective's premium. Notice that for positively homogeneous and cash-invariant risk measures, the approximation $\rho\left(\hat{\Phi}_{n}\right)$ can be expressed as

$$
\rho\left(\hat{\Phi}_{n}\right)=\hat{\sigma}_{n} \rho(\Phi)-\hat{\mu}_{n}
$$

where $\Phi$ refers to the standard normal df. The representation (1) provides an extremely simple tool for the approximation of the collective's premium $\rho\left(F_{n}\right)$, so that this (normal) approximation is quite popular in practice.

In this article we focus on how the error distance between $\rho\left(\hat{\Phi}_{n}\right)$ and $\rho\left(F_{n}\right)$ behaves asymptotically, relatively to $n$, as the number $n$ of clients increases. For a large class of distribution-invariant positively homogeneous and cash-invariant risk measures $\rho$ (in the sense of $[1,2,8,13])$ we will show that under fairly mild assumptions

$$
n^{1 / 2-\varepsilon} \frac{\rho\left(\hat{\Phi}_{n}\right)-\rho\left(F_{n}\right)}{n} \stackrel{a . s .}{\longrightarrow} 0, \quad n \rightarrow \infty
$$

for each $\varepsilon \in(0,1 / 2)$, and

$$
\operatorname{law}\left(n^{1 / 2} \frac{\rho\left(\hat{\Phi}_{n}\right)-\rho\left(F_{n}\right)}{n}\right) \stackrel{w}{\longrightarrow} \mathcal{N}, \quad n \rightarrow \infty
$$

for a certain normal distribution $\mathcal{N}$. Notice that $\rho_{g}\left(\hat{\Phi}_{n}\right) / n$ can be seen as an approximation of the individual premium $\rho_{g}\left(F_{n}\right) / n$.

Regarding $\rho$ we will at first focus on so called distortion risk measures introduced by Wang [36]. Given a distortion function $g$, i.e. a nondecreasing function $g$ on $[0,1]$ satisfying $g(0)=0$ and $g(1)=1$,

$$
\rho_{g}(F):=\int_{-\infty}^{0} g(F(t)) d t-\int_{0}^{\infty}[1-g(F(t))] d t
$$

provides a positively homogeneous distribution-invariant monetary risk measure on the class $\mathbb{F}_{g}$ of all df $F$ for which the integrals in (4) exists. It is subadditive (hence coherent) if and only if $g$ is concave (cf. [37]). As usual we refer to $\rho_{g}$ as distortion risk measure with distortion function $g$. If $g$ is a càdlàg function then we have the alternative representation $\rho_{g}(F)=-\int_{\mathbb{R}} x d g(F(x))$. Notice that most of the popular 
risk measures in practice can be represented as in (4), so that this class of risk measures has been studied extensively in the last decade, see [16, 19, 20, 36, 37, 38] and references cited therein. For instance, the Value-at-Risk $\operatorname{VaR}_{\alpha}$ at level $\alpha \in(0,1)$, the Average Value-at-Risk $\mathrm{AVaR}_{\alpha}$ at level $\alpha \in(0,1)$, and the Wang transform $W T_{\theta}$ with parameter $\theta \in \mathbb{R}$, correspond to the distortion functions $g(x)=\mathbb{1}_{[\alpha, 1]}(x), g(x)=(x / \alpha) \wedge 1$, and $g(x)=\Phi\left(\Phi^{-1}(x)-\theta\right)$, respectively. For details and other examples see, e.g., [19, 38]. In Section 2.1, we will establish (2) and (3) for $\rho=\rho_{g}$. We will see in particular that the goodness of the approximation of $\rho\left(F_{n}\right) / n$ by $\rho\left(\hat{\Phi}_{n}\right) / n$ is typically ruled by the goodness of the estimate $\hat{\mu}_{n} / n$ of $\mathbb{E}\left[X_{1}\right]$ (cf. Corollary 2.9 and its proof). In Section 2.2 we will see that the results of Section 2.1 can easily be extended to partially more general distribution-invariant risk measures. The key will be a robust representation (similar to results in [13]) of distribution-invariant coherent risk measures by distortion risk measures. In Section 3, we will illustrate our analysis by means of a numerical example, and in Section 4 we will give the proofs of our main results. Finally, in the Appendix A we will present some examples for risk measures that match the setting of Section 2.2.

\section{Results}

Let $X_{1}, X_{2}, \ldots$ be identically distributed square-integrable random variables on some probability space $(\Omega, \mathcal{F}, \mathbb{P})$. The random variable $X_{i}$ can be regarded as the claim of the $i$ th client, where a negative value corresponds to a payout to the client. We denote by $F_{n}$ the df of the total claim amount $S_{n}:=\sum_{i=1}^{n} X_{i}$, let $\hat{\mu}_{n}$ and $\hat{\sigma}_{n}$ be some estimates of $\mu_{n}:=\mathbb{E}\left[S_{n}\right]=n \mathbb{E}\left[X_{1}\right]$ and $\sigma_{n}:=\operatorname{Var}\left[S_{n}\right]^{1 / 2}$ (based on a sample of size $m_{n}$ ), and let $\Phi, \Phi_{n}$ and $\hat{\Phi}_{n}$ denote the standard normal df, the df of $\mathcal{N}_{\mu_{n}, \sigma_{n}^{2}}$ and the (random) df of $\mathcal{N}_{\hat{\mu}_{n}, \hat{\sigma}_{n}^{2}}$, respectively.

Throughout this article we will assume that the sequence $\left(X_{i}\right)$ is " $(\lambda, \gamma)$-Berry-Esseen". We say that $\left(X_{i}\right)$ is $(\lambda, \gamma)$-Berry-Esseen if there is some constant $c_{\lambda, \gamma}>0$ such that $\left\|G_{n}-\Phi\right\|_{\lambda} \leq c_{\lambda, \gamma} n^{-\gamma}$ for all $n \in \mathbb{N}$, where $G_{n}$ denotes the df of the random variable $Z_{n}:=\left(S_{n}-\mu_{n}\right) / \sigma_{n}$, and $\|\cdot\|_{\lambda}:=\left\|(\cdot) \phi_{\lambda}\right\|_{\infty}$ refers to the nonuniform sup-norm based on the weight function $\phi_{\lambda}(x):=(1+|x|)^{\lambda}, x \in \mathbb{R}$. In Example 2.1 we will give three examples for Berry-Esseen sequences. Two of them involve the notion of $\phi$ - and $\alpha$ mixing introduced in [18] and [32], respectively. Recall that $\left(X_{i}\right)$ is said to be $\phi$ - or $\alpha$-mixing according to as $\phi(n) \rightarrow 0$ or $\alpha(n) \rightarrow 0$, respectively, where

$$
\begin{aligned}
\phi(n) & :=\sup _{k \geq 1} \sup _{A \in \mathcal{F}_{1}^{k}, B \in \mathcal{F}_{n+k}^{\infty}}|\mathbb{P}[B \mid A]-\mathbb{P}[B]| \\
\alpha(n) & :=\sup _{k \geq 1} \sup _{A \in \mathcal{F}_{1}^{k}, B \in \mathcal{F}_{n+k}^{\infty}}|\mathbb{P}[A \cap B]-\mathbb{P}[A] \mathbb{P}[B]|
\end{aligned}
$$

with $\mathcal{F}_{n}^{m}:=\sigma\left(X_{i}: n \leq i \leq m\right) \subset \mathcal{F}$. For an overview on mixing conditions see, e.g., $[4,9]$. In [5], one can find several examples for strictly stationary $\phi$-mixing sequences (noting that $\phi$-mixing sequences are also $\beta$-mixing, cf. [4, (1.11)]). For examples for strictly stationary $\alpha$-mixing sequences see, e.g., [24]. 
Example 2.1 (i) If $X_{1}, X_{2}, \ldots$ are independent and $\mathbb{E}\left[\left|X_{1}\right|^{2+\delta}\right]<\infty$ for some $\delta>0$, then Michel's nonuniform Berry-Esseen inequality [25, Theorem 3] shows that $\left(X_{i}\right)$ is $(\lambda, \gamma)$-Berry-Esseen with $\lambda=2+\delta$ and $\gamma=\min \{\delta ; 1\} / 2$.

(ii) If $\left(X_{i}\right)$ is strictly stationary and $\phi$-mixing with mixing coefficients $\phi(n)$ satisfying $\phi(n) \leq c e^{-\kappa n}$ for all $n \in \mathbb{N}$ and some $c, \kappa>0, \mathbb{E}\left[\left|X_{1}\right|^{2+\delta}\right]<\infty$ for some $\delta \geq 1$, and $\lim _{n \rightarrow \infty} \sigma_{n} / \sqrt{n}=\sigma$ for some $\sigma>0$, then Schneider's nonuniform Berry-Esseen inequality [34, Theorem 4] shows that $\left(X_{i}\right)$ is $(\lambda, \gamma)$-Berry-Esseen for $\lambda=2+\delta$ and $\gamma \in(0,1 / 2)$. Notice that under the assumptions imposed on $\phi(n)$ we have that $\sigma_{n} / \sqrt{n}$ converges to $\sigma:=\left(\mathbb{V a r}\left[X_{1}\right]+2 \sum_{j=2}^{\infty} \mathbb{C o v}\left(X_{1}, X_{j}\right)\right)^{1 / 2}$, cf. Example 2.5(ii) below.

(iii) If $\left(X_{i}\right)$ is strictly stationary and $\alpha$-mixing with mixing coefficients $\alpha(n)$ satisfying $\sum_{n=1}^{\infty} \alpha(n)^{\varepsilon}<\infty$ for all $\varepsilon>0, \mathbb{E}\left[\left|X_{1}\right|^{q}\right]<\infty$ for all $q>0$, and $\lim _{n \rightarrow \infty} \sigma_{n} / \sqrt{n}=\sigma$ for some $\sigma>0$, then Hipp's nonuniform Berry-Esseen inequality [17, Theorem 2.5] shows that $\left(X_{i}\right)$ is $(\lambda, \gamma)$-Berry-Esseen for every $\lambda>0$ and $\gamma \in(0,1 / 2)$. Notice that under the assumptions imposed on $\alpha(n)$ we have that $\sigma_{n} / \sqrt{n}$ converges to $\sigma:=$ $\left(\mathbb{V a r}\left[X_{1}\right]+2 \sum_{j=2}^{\infty} \mathbb{C o v}\left(X_{1}, X_{j}\right)\right)^{1 / 2}$, cf. Example 2.5(iii) below.

\subsection{Distortion risk measures}

In this subsection we focus on distortion risk measures $\rho_{g}$ as introduced in (4). We first investigate the distance between $\rho_{g}\left(\Phi_{n}\right)$ and $\rho_{g}\left(F_{n}\right)$. As usual, $a \wedge b$ and $a \vee b$ denote the minimum and the maximum of $a, b \in \mathbb{R}$, respectively. Moreover we set $a^{+}:=a \vee 0$ and $a^{-}:=(-a) \vee 0$ for $a \in \mathbb{R}$.

Theorem 2.2 Let $g$ be a distortion function, and suppose that

(a) there are $L, \beta>0, k \in \mathbb{N}_{0}$, and $0=d_{0}<d_{1}<\ldots<d_{k+1}=1$, such that $g$ is Hölder- $\beta$-continuous with Hölder constant $L$ on each of the intervals $\left(d_{i}, d_{i+1}\right)$, $i=0, \ldots, k$,

(b) $\left(X_{i}\right)$ is $(\lambda, \gamma)$-Berry-Esseen for some $\gamma>0$ and $\lambda \geq 1$ satisfying $\lambda \beta>1$,

(c) $\lim _{n \rightarrow \infty} \sigma_{n} / \sqrt{n}=\sigma$ for some $\sigma>0$,

(d) $F_{n} \in \mathbb{F}_{g}$ and $\Phi_{n} \in \mathbb{F}_{g}$ for all $n \in \mathbb{N}$.

Then there is some finite constant $C=C_{k, L, \lambda, \gamma, \beta, \sigma}>0$, and some $n_{0} \in \mathbb{N}$ (depending only on the distribution of $\left.X_{1}\right)$, such that

$$
\left|\rho_{g}\left(F_{n}\right)-\rho_{g}\left(\Phi_{n}\right)\right| \leq C n^{1 / 2-(\beta \wedge 1) \gamma} \quad \forall n \geq n_{0}
$$

If $g$ is Hölder- $\beta$-continuous on all of $[0,1]$, i.e. if $k=0$, then in (b) the assumption $\lambda \geq 1$ can be replaced by $\lambda>0$, in the exponent on the right-hand side of (5) the expression $\beta \wedge 1$ can be replaced by $\beta$, and inequality (5) holds for all $n \in \mathbb{N}$.

The proof of Theorem 2.2 is relegated to Section 4.1. Assumption (d) in Theorem 2.2 is imposed to guarantee that the left-hand side of (5) is well-defined. In the following Examples 2.3-2.6 we illustrate conditions (a)-(d), respectively. 
Example 2.3 Condition (a) on $g$ in Theorem 2.2 is fulfilled for, e.g., $g_{1}(x)=\mathbb{1}_{[\alpha, 1]}(x)$ (Value-at-Risk), $g_{2}(x)=(x / \alpha) \wedge 1$ (Average Value-at-Risk) and $g_{3}(x)=\Phi\left(\Phi^{-1}(x)-\theta\right)$ (Wang transform) with any $\beta>0, \beta=1$ and $\beta \in(0,1)$, respectively.

Example 2.4 (i) If $X_{1}, X_{2}, \ldots$ are independent and $\mathbb{E}\left[\left|X_{1}\right|^{(2+\delta) \vee(1 / \beta)}\right]<\infty$ for some $\delta>0 \vee(1 / \beta-2)$, then according to Example $2.1(\mathrm{i})$ the sequence $\left(X_{i}\right)$ satisfies condition (b) in Theorem 2.2 with $\lambda=2+\delta$ and $\gamma=(\delta \wedge 1) / 2$.

(ii) If $\left(X_{i}\right)$ is strictly stationary and $\phi$-mixing with mixing coefficients $\phi(n)$ satisfying $\sum_{n=1}^{\infty} \phi(n)^{1 / 2}<\infty$, and $\mathbb{E}\left[\left|X_{1}\right|^{2+\delta}\right]<\infty$ for some $\delta>1 \vee(1 / \beta-2)$, then according to Example 2.1(ii) the sequence $\left(X_{i}\right)$ subject to condition (c) in Theorem 2.2 satisfies condition (b) in Theorem 2.2 with $\lambda=2+\delta$ and $\gamma \in(0,1 / 2)$.

(iii) If $\left(X_{i}\right)$ is strictly stationary and $\alpha$-mixing with mixing coefficients $\alpha(n)$ satisfying $\sum_{n=1}^{\infty} \alpha(n)^{\varepsilon}<\infty$ for every $\varepsilon>0$, and $\mathbb{E}\left[\left|X_{1}\right|^{q}\right]<\infty$ for every $q>0$, then according to Example 2.1(iii) the sequence $\left(X_{i}\right)$ subject to condition (c) in Theorem 2.2 satisfies condition (b) in Theorem 2.2 with $\lambda>1 / \beta$ and $\gamma \in(0,1 / 2)$.

Example 2.5 (i) If $X_{1}, X_{2}, \ldots$ are independent then the sequence $\left(X_{i}\right)$ clearly satisfies condition (c) in Theorem 2.2 for $\sigma:=\operatorname{Var}\left[X_{1}\right]^{1 / 2}$.

(ii) If $\left(X_{i}\right)$ is strictly stationary and $\phi$-mixing with mixing coefficients $\phi(n)$ satisfying $\sum_{n=1}^{\infty} \phi(n)^{1 / 2}<\infty$, and $\mathbb{E}\left[\left|X_{1}\right|^{2+\delta}\right]<\infty$ for some $\delta>0$, then the sequence $\left(X_{i}\right)$ satisfies condition (c) in Theorem 2.2 for $\sigma:=\left(\mathbb{V a r}\left[X_{1}\right]+2 \sum_{j=2}^{\infty} \mathbb{C o v}\left(X_{1}, X_{j}\right)\right)^{1 / 2}$, see the proof of Lemma 4.2.2 in [29].

(iii) If $\left(X_{i}\right)$ is strictly stationary and $\alpha$-mixing with mixing coefficients $\alpha(n)$ satisfying $\sum_{n=1}^{\infty} \alpha(n)^{\delta /(2+\delta)}<\infty$ for some $\delta>0$, and $\mathbb{E}\left[\left|X_{1}\right|^{2+\delta}\right]<\infty$, then the sequence $\left(X_{i}\right)$ satisfies condition (c) in Theorem 2.2 for $\sigma:=\left(\mathbb{V a r}\left[X_{1}\right]+2 \sum_{j=2}^{\infty} \mathbb{C o v}\left(X_{1}, X_{j}\right)\right)^{1 / 2}$, see [27, Corollary 1.1].

Example 2.6 If there are $0<x_{0} \leq x_{1}<1$ so that $g(x)=0$ for $x \in\left[0, x_{0}\right)$ and $g(x)=1$ for $x \in\left(x_{1}, 1\right]$ (which is the case, e.g., for $g_{1}$ in Example 2.3), then condition (d) in Theorem 2.2 is always fulfilled. Moreover, if $F_{1} \in \mathbb{F}_{g}$ and $\Phi \in \mathbb{F}_{g}$ then condition (d) is also fulfilled whenever $g$ is concave (which is the case, e.g., for $g_{2}$ and $g_{3}$ in Example 2.3). Indeed in this case $\rho_{g}$ may be viewed as a Choquet integral w.r.t. the set function $g(\mathbb{P}[\cdot])$ (cf. [13]), and the space of random variables on $(\Omega, \mathcal{F}, \mathbb{P}$ ) with df in $\mathbb{F}_{g}$ is a vector subspace of the space of all random variables on $(\Omega, \mathcal{F}, \mathbb{P})$ (cf. [7]). $\diamond$

We emphasize that in the setting of Example 2.4(i), for each of the risk measures $\rho_{g_{i}}$ in Example 2.3 the right-hand side of (5) is strictly positive and bounded below. This is also true for every coherent distortion risk measures since the concavity of $g$ implies $\beta \leq 1$. For $g_{1}$ and $g_{2}$ in Example 2.3, and for all other distortion functions $g$ for which we can choose $\beta=1$, the right-hand side of (5) can even be chosen to be constant (provided $\gamma \geq 1 / 2$, e.g., if $\delta \geq 1$ in the setting of Example 2.4). So in this case the 
relative error $\left|\rho_{g}\left(F_{n}\right)-\rho_{g}\left(\Phi_{n}\right)\right| / n$ converges at least at rate 1 . On the other hand, in general, no matter how small $\beta, \gamma>0$ are, the relative error converges always faster than at rate $1 / 2$.

As indicated in the introduction, in practice one is actually interested in statements as (2) where $\mu_{n}$ and $\sigma_{n}$ are estimated by some suitable estimators $\hat{\mu}_{n}$ and $\hat{\sigma}_{n}$ based on random variables $Y_{1}, \ldots, Y_{m_{n}}$ having the same distribution as $X_{1}$. We will assume throughout the rest of this section that

$$
n^{-(1 / 2+\varepsilon)}\left(\hat{\mu}_{n}-\mu_{n}\right) \stackrel{a . s .}{\longrightarrow} 0
$$

for some $\varepsilon \in(0,1 / 2)$, and that

$$
n^{-1 / 2}\left(\hat{\sigma}_{n}-\sigma_{n}\right) \stackrel{a . s .}{\longrightarrow} 0 .
$$

The following two examples show that the conditions (6)-(7) are not too restrictive. Throughout theses examples we shall assume $m_{n} \sim n$, i.e. that there is some constant $c>0$ such that $m_{n} / n \rightarrow c$ as $n \rightarrow \infty$.

Example 2.7 (i) If $Y_{1}, Y_{2}, \ldots$ are independent, then (6) holds for $\hat{\mu}_{n}:=\frac{n}{m_{n}} \sum_{i=1}^{m_{n}} Y_{i}$ and every $\varepsilon \in(0,1 / 2)$ by the Marcinkiewicz-Zygmund SLLN (cf. [11, 35]) and Slutzky's lemma.

(ii) If $\left(Y_{i}\right)$ is strictly stationary and $\phi$-mixing with mixing coefficients $\phi(n)$ satisfying $\sum_{n=1}^{\infty} \phi\left(2^{n}\right)^{1 / 2}<\infty$, then (6) holds for $\hat{\mu}_{n}:=\frac{n}{m_{n}} \sum_{i=1}^{m_{n}} Y_{i}$ and every $\varepsilon \in(0,1 / 2)$ by Peligrad's Marcinkiewicz-Zygmund type SLLN for strictly stationary $\phi$-mixing sequences [26, Theorem 2] and Slutzky's lemma.

(iii) If $\left(Y_{i}\right)$ is strictly stationary and $\alpha$-mixing with mixing coefficients $\alpha(n)$ satisfying $\sum_{n=1}^{\infty} n^{(1-4 \varepsilon) /(2 \varepsilon)} \alpha(n)<\infty$ for some $\varepsilon \in(0,1 / 2)$, then (6) holds for $\hat{\mu}_{n}:=\frac{n}{m_{n}} \sum_{i=1}^{m_{n}} Y_{i}$ (with the same $\varepsilon$ ) by Rio's Marcinkiewicz-Zygmund type SLLN for strictly stationary $\alpha$-mixing sequences (cf. [31, Theorem 1 along with (1.10) with $r=2$ ]) and Slutzky's lemma.

Example 2.8 (i) If $X_{1}, X_{2}, \ldots$ are independent and $Y_{1}, Y_{2}, \ldots$ are independent, then (7) holds for $\hat{\sigma}_{n}:=\left(\frac{n}{m_{n}} \sum_{i=1}^{m_{n}}\left(Y_{i}-\frac{1}{m_{n}} \sum_{i=1}^{m_{n}} Y_{i}\right)^{2}\right)^{1 / 2}$ by the classical Kolmogorov SLLN and Slutzky's lemma.

(ii) If the sequences $\left(X_{i}\right)$ and $\left(Y_{i}\right)$ coincide in law, $\left(X_{i}\right)$ is strictly stationary and $\alpha$-mixing with mixing coefficients $\alpha(n)$ satisfying $\sum_{n=1}^{\infty} \alpha(n)^{\delta /(2+\delta)}<\infty$ for some $\delta>0$, and $\mathbb{E}\left[\left|X_{1}\right|^{2+\delta}\right]<\infty$, then (7) holds for $\hat{\sigma}_{n}:=\sqrt{\frac{\pi}{2}} \frac{\sqrt{n}}{\log m_{n}} \sum_{i=1}^{m_{n}} \frac{1}{\sqrt{i}} \mid \frac{1}{i} \sum_{j=1}^{i} Y_{j}-$ $\frac{1}{m_{n}} \sum_{i=1}^{m_{n}} Y_{i} \mid$ by Corollary 1.1 in [27] and Slutzky's lemma.

Corollary 2.9 (Rate of convergence) Suppose that the assumptions of Theorem 2.2 and the conditions (6) and (7) hold. Then we have (2) with $\rho=\rho_{g}$ (and $\varepsilon$ as in (6)), i.e.

$$
n^{1 / 2-\varepsilon} \frac{\rho_{g}\left(\hat{\Phi}_{n}\right)-\rho_{g}\left(F_{n}\right)}{n} \stackrel{a . s .}{\longrightarrow} 0, \quad n \rightarrow \infty .
$$


Proof By the triangle inequality, the representation (1) for $\rho_{g}\left(\Phi_{n}\right)$ (recall that $\rho_{g}$ is the positively homogeneous and cash-invariant), the analogous representation for $\rho_{g}\left(\Phi_{n}\right)$, and (5), we have

$$
\begin{aligned}
\frac{\left|\rho_{g}\left(\hat{\Phi}_{n}\right)-\rho_{g}\left(F_{n}\right)\right|}{n} & \leq \frac{\left|\rho_{g}\left(\hat{\Phi}_{n}\right)-\rho_{g}\left(\Phi_{n}\right)\right|}{n}+\frac{\left|\rho_{g}\left(\Phi_{n}\right)-\rho_{g}\left(F_{n}\right)\right|}{n} \\
& \leq \frac{\left|\hat{\sigma}_{n}-\sigma_{n}\right|\left|\rho_{g}(\Phi)\right|+\left|\mu_{n}-\hat{\mu}_{n}\right|}{n}+C n^{-1 / 2-(\beta \wedge 1) \gamma}
\end{aligned}
$$

with $\Phi$ the standard normal df. By (6) we have that $\left|\mu_{n}-\hat{\mu}_{n}\right| / n$ converges to 0 at least at rate $1 / 2-\varepsilon$. By $(7)$ we have that $\left|\hat{\sigma}_{n}-\sigma_{n}\right| / n$ converges to 0 at least at rate $1 / 2$. Moreover we obviously have that the latter summand on the right-hand side of (8) converges to 0 at rate $1 / 2+(\beta \wedge 1) \gamma$. The statement of Corollary 2.9 then follows from (8).

Corollary 2.9 and its proof show that the rate of the convergence of $\left(\rho_{g}\left(\Phi_{n}\right)-\rho_{g}\left(F_{n}\right)\right) / n$ to 0 is not smaller than the rate of the convergence of the estimator $\hat{\mu}_{n} / n$ to $\mathbb{E}\left[X_{1}\right]$. We realize that in this case the relative approximation of $\rho_{g}\left(F_{n}\right)$ by $\rho_{g}\left(\Phi_{n}\right)$ is asymptotically not worse than the estimation of the mean $\mathbb{E}\left[X_{1}\right]$. We can also deduce that if

$$
\operatorname{law}\left(\sqrt{n} \frac{\hat{\mu}_{n}-\mu_{n}}{n}\right) \stackrel{w}{\longrightarrow} \mathcal{N}, \quad n \rightarrow \infty
$$

for some distribution $\mathcal{N}$ on $(\mathbb{R}, \mathcal{B}(\mathbb{R}))$, then the asymptotic error distribution $\mathcal{N}$ in (3) is given by the same $\mathcal{N}$, cf. Corollary 2.10. Notice that assumption (9) is not too restrictive. Indeed: For $\hat{\mu}_{n}:=\frac{n}{m_{n}} \sum_{i=1}^{m_{n}} Y_{i}$ the Examples 2.1 and 2.5 provide standard settings where (9) holds for $\mathcal{N}=\mathcal{N}_{0, \sigma^{2}}$ (just replace $\left(X_{i}\right)$ by $\left(Y_{i}\right)$ ), and Example 2.8 shows that in some situations it is even possible to estimate the asymptotic standard deviation $\sigma$ consistently.

Corollary 2.10 (Asymptotic error distribution) Suppose the assumptions of Theorem 2.2 and the conditions (7) and (9) hold. Then we have (3) with $\rho=\rho_{g}$, i.e.

$$
\operatorname{law}\left(\sqrt{n} \frac{\rho_{g}\left(\hat{\Phi}_{n}\right)-\rho_{g}\left(F_{n}\right)}{n}\right) \stackrel{w}{\longrightarrow} \mathcal{N}, \quad n \rightarrow \infty .
$$

Proof The statement of Corollary 2.10 follows immediately from (8) (without absolute values and with " $\leq$ " replaced by "="), condition (9) and Slutzky's lemma.

Remark 2.11 It is worth mentioning that the normal approximation makes sense also for parametric models for the distribution of $X_{1}$ since the convolution is typically non-determinable. For parametric models the estimators $\hat{\mu}_{n}$ and $\hat{\sigma}_{n}$ might be chosen according to the particular model (and might possess partially better statistical properties compared to the nonparametric estimators considered in Examples 2.7 and 2.8), but the asymptotic behavior of the estimators exploited in the corollaries is typically the same. 


\subsection{Extension to partially more general distribution-invariant risk measures}

In this section, we focus on distribution-invariant coherent risk measures $\rho$ that are not necessarily distortion risk measures but that can be robustly represented by distortion risk measures (more precisely by concave distortion functions). That is, we suppose that there exists a set $\mathcal{G}_{\rho}$ of concave distortion functions such that

$$
\rho(F)=\sup _{g \in \mathcal{G}_{\rho}} \rho_{g}(F)
$$

for every admissible df $F$, where $\rho_{g}$ denotes the distortion risk measure with distortion function $g$ (cf. (4)). So we have in particular

$$
\left|\rho\left(F_{n}\right)-\rho\left(\Phi_{n}\right)\right|=\left|\sup _{g \in \mathcal{G}_{\rho}} \rho_{g}\left(F_{n}\right)-\sup _{g \in \mathcal{G}_{\rho}} \rho_{g}\left(\Phi_{n}\right)\right| \leq \sup _{g \in \mathcal{G}_{\rho}}\left|\rho_{g}\left(F_{n}\right)-\rho_{g}\left(\Phi_{n}\right)\right| .
$$

Now, observe that the constant $C$ in the basic Theorem 2.2 depends only on $\beta$ and $L$ (other properties of $g$ do not play any role). Therefore the result of Theorem 2.2 carries over to $\rho$ in (10) if, for some $\beta>0$ fixed, all distortion functions $g \in \mathcal{G}_{\rho}$ are Hölder- $\beta$-continuous with a common Hölder constant $L$. Of course, in this case the Corollaries 2.9 and 2.10 carry over to $\rho$ in (10) as well. In Theorem 2.12 we will see that under some assumptions on $\rho$ the robust representation (10) holds. In Lemma 2.14 we will obtain a criterion to check whether the distortion functions in $\mathcal{G}_{\rho}$ are all Hölder- $\beta$-continuous with a common Hölder constant $L$.

We first discuss the existence of the representation (10). Such a representation result is already known from [13] but we will give a generalized version; notice that (10) differs from the celebrated Kusuoka representation (see [23], and [28] for an extension). The basic setting is the following. Let $(\Omega, \mathcal{F}, \mathbb{P})$ be a probability space that is rich enough to support a random variable with continuous distribution (which is equivalent to $(\Omega, \mathcal{F}, \mathbb{P})$ being atomless in the sense of $[13$, Definition A.26]). Let $\mathfrak{X}$ be a subspace of $\mathcal{L}^{1}(\Omega, \mathcal{F}, \mathbb{P})$ such that $\mathcal{L}^{\infty}(\Omega, \mathcal{F}, \mathbb{P}) \subset \mathfrak{X}$ and $X \wedge Y, X \vee Y \in \mathfrak{X}$ for all $X, Y \in \mathfrak{X}$, and let $\mathfrak{X}_{+}$be the class of all nonnegative random variables of $\mathfrak{X}$. We denote by $\mathbb{F}$ the set of all df $F_{X}$ of random variables $X \in \mathfrak{X}$, and we consider a distributioninvariant coherent risk measure $\rho: \mathbb{F} \rightarrow \mathbb{R}$. Finally we denote by $B_{x}$ any random variable distributed according to the Bernoulli distribution with parameter $x$. Recall that every nondecreasing concave function on $[0,1]$ is continuous on $(0,1]$.

Theorem 2.12 (Robust representation) If the cutting condition

$$
\lim _{n \rightarrow \infty} \rho\left(F_{-\lambda(X-n)^{+}}\right)=0 \quad \forall X \in \mathfrak{X}_{+}, \lambda>0
$$

holds then there is some set $\mathcal{G}_{\rho}$ of concave distortion functions satisfying (10) for all $F \in \mathbb{F}$. If in addition

$$
\lim _{x \rightarrow 0+} \rho\left(F_{-B_{x}}\right)=0
$$

then all functions $g \in \mathcal{G}_{\rho}$ are continuous at 0 (and therefore continuous on $[0,1]$ ). 
The proof of Theorem 2.12 is relegated to Section 4.2. In Lemma A.1 in the Appendix A we will see that if $\mathfrak{X}$ is chosen to be an Orlicz space with continuous Young function, then every coherent risk measure $\rho$ satisfies the conditions (11)-(12). In the Appendix A we will also give examples of particular coherent risk measures on Orlicz spaces satisfying (11)-(12). Further, we note that it is an easy consequence of (14) below that condition (12) ensures that the functions $g \in \mathcal{G}_{\rho}$ are continuous at 0 (i.e. that the second part of Theorem 2.12 holds true), where

$$
\widetilde{g}_{\rho}(x):=\sup _{g \in \mathcal{G}_{\rho}} g(x), \quad x \in[0,1] .
$$

Notice that the function $\widetilde{g}_{\rho}$ is again a distortion function which we refer to as distortion function associated with $\rho$. We reasonably denote the corresponding distortion risk measure by $\rho_{\widetilde{g}_{\rho}}$.

Remark 2.13 (i) If $\rho$ is known to be a distortion risk measure (as defined in (4)) then its distortion function $g_{\rho}$ can clearly be recovered from $\rho$ by $g_{\rho}(x)=\rho\left(F_{-} B_{x}\right)$, $x \in[0,1]$, where as above $B_{x}$ refers to any random variable distributed according to the Bernoulli distribution with parameter $x$.

(ii) If the risk measure $\rho$ satisfies (10) for all $F \in \mathbb{F}$, then $\rho$ will degenerate to a distortion risk measure if and only if $\rho=\rho_{\widetilde{g}_{\rho}}$ with $\widetilde{g}_{\rho}$ as in (13). The necessity is obvious, and the sufficiency can be seen as follows. If $\rho$ is a distortion risk measure then according to (i) its distortion function $g_{\rho}$ is given by $g_{\rho}(x)=\rho\left(F_{-B_{x}}\right), x \in[0,1]$. The identity (14) in Lemma 2.14 below then shows that $g_{\rho}$ must coincide with the distortion function $\widetilde{g}_{\rho}$ associated with $\rho$.

The following lemma gives a criterion for the distortion functions $g \in \mathcal{G}_{\rho}$ to be all Hölder- $\beta$-continuous with a common Hölder constant.

Lemma 2.14 Suppose (10) holds for all $F \in \mathbb{F}$ for some set $\mathcal{G}_{\rho}$ of concave distortion functions, and let $\widetilde{g}_{\rho}$ denote the distortion function associated with $\rho$ (cf. (13)). Then we have

$$
\widetilde{g}_{\rho}(x)=\rho\left(F_{-B_{x}}\right), \quad x \in[0,1],
$$

where as above $B_{x}$ refers to any random variable distributed according to the Bernoulli distribution with parameter $x$. Moreover, if every $g \in \mathcal{G}_{\rho}$ is continuous at 0 , and if for some $\beta>0$ the condition

$$
L:=\sup _{x \in(0,1]} \frac{\widetilde{g}_{\rho}(x)}{x^{\beta}}<\infty
$$

holds, then every $g \in \mathcal{G}_{\rho}$ is Hölder- $\beta$-continuous with Hölder constant $L$.

The proof of Lemma 2.14 is relegated to Section 4.3. Condition (15) provides a transparent condition in order to obtain a common Hölder constant. Equation (14) in turn provides a useful tool for the calculation of $\widetilde{g}_{\rho}(x)$; the values $\widetilde{g}_{\rho}(x), x \in[0,1]$, are needed for checking condition (15). In the Appendix A, we will give examples for distribution-invariant coherent risk measures satisfying condition (15). Examples are Haezendonck risk measures and risk measures based on one-sided moments. 


\section{Numerical example}

In this section, we are going to illustrate Theorem 2.2 as well as the Corollaries 2.9 and 2.10 by means of a numerical example. We consider an i.i.d. insurance collective of size $n$ and assume that the individual claim df $F_{1}$ is given by the Gamma df on $\mathbb{R}_{-}$ (i.e., $\left.-X_{1} \sim \Gamma(a, b)\right)$ with parameters $a=4$ and $b=0.004$. We note that the Gamma distribution is often used in insurance practice to model small to moderate individual claims (as for instance in third party car insurance or home contents insurance). The mean and the standard deviation of $F_{1}$ are given by $-a / b=-1^{\prime} 000$ and $\sqrt{a / b^{2}}=500$. The $n$-fold convolution $F_{n}$ of $F_{1}$ is known to be the Gamma df on $\mathbb{R}_{-}$with parameters $n a=4 n$ and $b=0.004$, and the mean and the standard deviation of $F_{n}$ are given by $-1^{\prime} 000 n$ and $\sqrt{n a / b^{2}}=500 \sqrt{n}$, respectively. As underlying "premium principle" we consider the Value-at-risk at level $\alpha=0.05$ which is the distortion risk measure $\rho_{g}$ with distortion function $g=\mathbb{1}_{[\alpha, 1]}$.

As already mentioned subsequent to Example 2.6, Theorem 2.2 indicates that under some assumptions (which are fulfilled in the present setting) the right-hand side of (5) can be chosen to be constant. The second row in Figure 1 shows that in our example the difference $\rho\left(\Phi_{n}\right)-\rho\left(F_{n}\right)$ is indeed nearly constant as $n$ increases. That is, relative to the number of clients $n$, the approximation of $\rho\left(F_{n}\right)$ by $\rho\left(\Phi_{n}\right)$ is of order $n^{-1}$. Hence the approximation is pretty good, see also the first row in Figure 1.

However, in practice the exact mean and the exact standard deviations are unknown and have be to estimated. So, in fact, for practical purposes it is more interesting to study the asymptotic behavior of the difference $\rho\left(\hat{\Phi}_{n}\right)-\rho\left(F_{n}\right)$. The Corollaries 2.9 and 2.10 show that the approximation of $\rho\left(F_{n}\right)$ by $\rho\left(\hat{\Phi}_{n}\right)$ relative to the number of clients $n$ is of order $n^{-1 / 2+\varepsilon}$ and that the relative difference $\left(\rho\left(\hat{\Phi}_{n}\right)-\rho\left(F_{n}\right)\right) / n$ weighted by $n^{1 / 2}$ is asymptotically normal. Figure 2 shows that in our example the relative error $\left(\rho\left(\Phi_{n}\right)-\rho\left(F_{n}\right)\right) / n$ indeed converges to 0 , and that the relative error weighted by $n^{1 / 2}$ is indeed subject to a random fluctuation. Figure 2 also shows that the approximation of $\rho\left(F_{n}\right)$ by $\rho\left(\hat{\Phi}_{n}\right)$ is "aggressive", which might be due to the fact that the tail of the Gamma distribution is "heavier" than the tail of a normal distribution.

\section{Proofs}

\subsection{Proof of Theorem 2.2}

The proof of Theorem 2.2 relies on the following lemma which involves the leftcontinuous inverse $H^{\leftarrow}(x):=\inf \{t \in \mathbb{R}: H(t) \geq x\}$ of a df $H$.

Lemma 4.1 ([38, Theorem 2]) Let $g$ be a distortion function, and suppose that

(i) there are $\beta, L>0, k \in \mathbb{N}_{0}$, and $0=d_{0}<d_{1}<\ldots<d_{k+1}=1$, such that $g$ is Hölder- $\beta$-continuous with Hölder constant $L$ on each of the intervals $\left(d_{i}, d_{i+1}\right)$, $i=0, \ldots, k$,

(ii) $H, H_{1}, H_{2}, \ldots \in \mathbb{F}_{g}$, 
(iii) $H$ is differentiable at $H^{\leftarrow}\left(d_{i}\right)$, and $H^{\prime}\left(H^{\leftarrow}\left(d_{i}\right)\right)>0$, for $i=1, \ldots, k$,

(iv) $\left\|H_{n}-H\right\|_{\infty} \rightarrow 0$.

For every $\lambda \geq 1$ satisfying $\lambda \beta>1$ there is a finite constant $c=c_{k, H, L, \lambda \beta}>0$, and some $n_{0} \in \mathbb{N}$ (for which $\left\|H_{n}-H\right\|_{\infty}$ is sufficiently small for all $n \geq n_{0}$ ), such that

$$
\left|\rho_{g}\left(H_{n}\right)-\rho_{g}(H)\right| \leq c\left\|H_{n}-H\right\|_{\lambda}^{\beta \wedge 1} \quad \forall n \geq n_{0} .
$$

If $g$ is Hölder- $\beta$-continuous on all of $[0,1]$, i.e. if $k=0$, then condition (iv) can be skipped, the assumption $\lambda \geq 1$ can be replaced by $\lambda>0$, in (16) the exponent $\beta \wedge 1$ can be replaced by $\beta$, and inequality (16) holds for all $n \in \mathbb{N}$.

Notice that under the assumptions of Lemma 4.1, the expression $\left\|H_{n}-H\right\|_{\lambda}$ might be infinite when $\lambda>1$. Also, $\left\|H_{n}-H\right\|_{\lambda} \rightarrow 0$ implies condition (iv).

In order to prove Theorem 2.2 we set $Z_{n}:=\left(S_{n}-\mu_{n}\right) / \sigma_{n}$ and write $N_{n}$ for any random variable distributed according to the normal law $\mathcal{N}_{\mu_{n}, \sigma_{n}^{2}}$. We further define $Z:=\left(N_{n}-\mu_{n}\right) / \sigma_{n}$ which is $\mathcal{N}_{0,1}$-distributed. As $\rho_{g}$ is distribution-invariant, we may and do use without risk of ambiguity $\rho_{g}(Y)$ and $\rho_{g}\left(F_{Y}\right)$ as synonyms, where $Y$ is any admissible random variable. By the positive homogeneity and the translation invariance of $\rho_{g}$ we obtain

$$
\begin{aligned}
\left|\rho_{g}\left(F_{n}\right)-\rho_{g}\left(\Phi_{n}\right)\right| & =\left|\rho_{g}\left(S_{n}\right)-\rho_{g}\left(N_{n}\right)\right| \\
& =\left|\rho_{g}\left(\sigma_{n} Z_{n}+\mu_{n}\right)-\rho_{g}\left(\sigma_{n} Z+\mu_{n}\right)\right| \\
& =\left|\sigma_{n} \rho_{g}\left(Z_{n}\right)-\mu_{n}-\left(\sigma_{n} \rho_{g}(Z)-\mu_{n}\right)\right| \\
& =\sigma_{n}\left|\rho_{g}\left(Z_{n}\right)-\rho_{g}(Z)\right| \\
& =\sigma_{n}\left|\rho_{g}\left(G_{n}\right)-\rho_{g}(\Phi)\right|,
\end{aligned}
$$

where $G_{n}$ denotes the df of $Z_{n}$, and $\Phi$ is the standard normal df. Now, we intend to apply Lemma 4.1. We are going to show that the assumptions (i)-(iv) of Lemma 4.1 are fulfilled for $H:=\Phi$ and $H_{n}:=G_{n}$. Condition (i) is ensured by assumption (a) of Theorem 2.2, the validity of condition (ii) was already discussed subsequent to Theorem 2.2, and condition (iii) is obvious since $\Phi$ is strictly increasing and smooth. By assumption (b) of Theorem 2.2 we moreover have

$$
\left\|G_{n}-\Phi\right\|_{\lambda} \leq c_{\lambda, \gamma} n^{-\gamma} \quad \forall n \in \mathbb{N}
$$

for some constant $c_{\lambda, \gamma}>0$, so that condition (iv) of Lemma 4.1 holds too. Therefore the lemma implies

$$
\left|\rho_{g}\left(G_{n}\right)-\rho_{g}(\Phi)\right| \leq c_{k, \Phi, L, \lambda \beta}\left\|G_{n}-\Phi\right\|_{\lambda}^{\beta \wedge 1} \quad \forall n \geq n_{0}
$$

for some constant $c_{k, \Phi, L, \lambda \beta}>0$. Along with assumption (c) of Theorem 2.2 as well as (17) and (18), this implies (5). 


\subsection{Proof of Theorem 2.12}

The verification of the second part of Theorem 2.12 was already given subsequent to Theorem 2.12. The proof of the first part relies on the following lemma.

Lemma 4.2 Condition (11) implies

$$
\rho\left(F_{X}\right)=\sup _{m \in \mathbb{N}} \inf _{k \in \mathbb{N}} \rho\left(F_{[X+\wedge k]-[X-\wedge m]}\right) \quad \forall X \in \mathfrak{X} .
$$

Proof In view of Proposition 6.6 in [22], condition (11) allows to apply Lemma 6.5 in [22]. Assertion .1 of this lemma gives $\sup _{m \in \mathbb{N}} \rho\left(F_{X^{+-}-\left[X^{-} \wedge m\right]}\right)=\rho\left(F_{X}\right)$, and assertion .2 of this lemma gives $\inf _{k \in \mathbb{N}} \rho\left(F_{\left[X^{+} \wedge k\right]-\left[X^{-} \wedge m\right]}\right)=\rho\left(F_{X^{+}-\left[X^{-} \wedge m\right]}\right)$ for every $m \in \mathbb{N}$. The statement of Lemma 4.2 is obvious now.

We will combine Lemma 4.2 with known representation results (mentioned above) for distribution-invariant coherent risk measures defined on the set $\mathbb{F}^{\infty}$ of all df $F_{X}$ of random variables $X \in \mathcal{L}^{\infty}(\Omega, \mathcal{F}, \mathbb{P})$ in order to prove Theorem 2.12.

Proof (of Theorem 2.12) Possibly changing to a suitable probability space, we may assume that $L^{2}(\Omega, \mathcal{F}, \mathbb{P})$ is separable. Then in the specified setting, Corollary 4.72 in [13] along with Theorem 2.1 in [21] yields the existence of some set $\mathcal{G}$ of concave distortion functions such that (10) holds for all $F \in \mathbb{F}^{\infty}$. Now set

$$
\widetilde{\rho}(F):=\sup _{g \in \mathcal{G}} \rho_{g}(F), \quad F \in \mathbb{F} .
$$

We are going to show that $\widetilde{\rho}$ provides a distribution-invariant coherent risk measure on $\mathbb{F}$ which coincides with $\rho$. We will proceed in two steps: First we will show that $\widetilde{\rho}$ is well-defined and defines a distribution-invariant coherent risk measure on $\mathbb{F}$ (which obviously satisfies $\widetilde{\rho}=\rho$ on $\left.\mathbb{F}^{\infty}\right)$. Second we will show that $\widetilde{\rho}=\rho$ on $\mathbb{F}$.

Step 1. If we can show that $\rho_{g}(F) \in \mathbb{R}$ (for all $g \in \mathcal{G}$ ) and $\sup _{g \in \mathcal{G}} \rho_{g}(F)<\infty$ for all $F \in \mathbb{F}$, then it follows easily that $\widetilde{\rho}$ defines a distribution-invariant coherent risk measure on $\mathbb{F}$, since every distortion risk measure $\rho_{g}$ with concave distortion function $g$ is coherent and distribution-invariant. Of course, in view of (4), the mentioned conditions hold if we can show

$$
\sup _{g \in \mathcal{G}} \int_{-\infty}^{0} g(F(t)) d t \leq \rho\left(F_{-X^{-}}\right) \quad \text { and } \quad \forall g \in \mathcal{G}: \quad \int_{0}^{\infty}[1-g(F(t))] d t<\infty
$$

for all $F \in \mathbb{F}$. To verify the first statement in (19), we pick $X \in \mathfrak{X}$ (corresponding to $\left.F_{X} \in \mathbb{F}\right)$. For every $g \in \mathcal{G}$ we have

$$
g\left(F_{-X^{-}}(t)\right) \leq \liminf _{m \rightarrow \infty} g\left(F_{-\left[X^{-} \wedge m\right]}(t)\right)
$$

at every continuity point $t<0$ of $F_{-X^{-}}$, since $g$ as a concave function is lower semicontinuous. Using this and applying Fatou's lemma, we obtain

$$
\sup _{g \in \mathcal{G}} \int_{-\infty}^{0} g\left(F_{X}(t)\right) d t \leq \sup _{g \in \mathcal{G}} \int_{-\infty}^{0} g\left(F_{-X^{-}}(t)\right) d t
$$




$$
\begin{aligned}
& \leq \sup _{g \in \mathcal{G}} \int_{-\infty}^{0} \liminf _{m \rightarrow \infty} g\left(F_{-\left[X^{-} \wedge m\right]}(t)\right) d t \\
& \leq \sup _{g \in \mathcal{G}} \liminf _{m \rightarrow \infty} \int_{-\infty}^{0} g\left(F_{-\left[X^{-} \wedge m\right]}(t)\right) d t \\
& =\sup _{g \in \mathcal{G}} \liminf _{m \rightarrow \infty} \rho_{g}\left(F_{-\left[X^{-} \wedge m\right]}\right) \\
& \leq \liminf _{m \rightarrow \infty} \rho\left(F_{-\left[X^{-} \wedge m\right]}\right) \\
& \leq \rho\left(F_{-X^{-}}\right) .
\end{aligned}
$$

Hence the first statement in (19) holds indeed. To verify the second statement in (19), we pick $X \in \mathfrak{X}$ (corresponding to $F_{X} \in \mathbb{F}$ ) and we denote by $g^{\prime}$ the right-sided derivative of $g$. As $g$ is a nondecreasing concave function, it is continuous on $(0,1]$, so that

$$
1-g\left(F_{X}(t)\right)=g(1)-g\left(F_{X}(t)\right) \leq g^{\prime}\left(F_{X}\left(t_{0}\right)\right)\left[1-F_{X}(t)\right] \quad \forall t \geq t_{0},
$$

for any $t_{0}>0$ such that $F_{X}\left(t_{0}\right)>0$. Moreover, the integral $\int_{0}^{\infty}\left[1-F_{X}(t)\right] d t$ exists since $X \in \mathcal{L}^{1}(\Omega, \mathcal{F}, \mathbb{P})$. Hence,

$$
\int_{0}^{\infty}\left[1-g\left(F_{X}(t)\right)\right] d t \leq \int_{0}^{t_{0}}\left[1-g\left(F_{X}(t)\right)\right] d t+g^{\prime}\left(F_{X}\left(t_{0}\right)\right) \int_{t_{0}}^{\infty}\left[1-F_{X}(t)\right] d t<\infty .
$$

This shows that the second statement in (19) holds, too.

Step 2. Condition (11) on $\rho$ ensures that the right-hand side of

$$
0 \leq \widetilde{\rho}\left(F_{-\lambda(X-r)^{+}}\right) \leq \rho\left(F_{-\lambda(X-r)^{+}}\right)
$$

converges to 0 , as $r \rightarrow \infty$, for every $X \in \mathfrak{X}_{+}$and $\lambda>0$. Therefore condition (11) is also fulfilled by $\widetilde{\rho}$, and Lemma 4.2 applied to $\widetilde{\rho}$ implies $\rho=\widetilde{\rho}$ on $\mathbb{F}$.

\subsection{Proof of Lemma 2.14}

Due to (10), Remark 2.13(i) and the definition of $\widetilde{g}_{\rho}$, we have

$$
\rho\left(F_{-B_{x}}\right)=\sup _{g \in \mathcal{G}_{\rho}} \rho_{g}\left(F_{-B_{x}}\right)=\sup _{g \in \mathcal{G}_{\rho}} g(x)=\widetilde{g}_{\rho}(x), \quad x \in[0,1],
$$

i.e. (14) holds.

In order to prove the second part of Lemma 2.14, we let $0<x<x^{\prime} \leq 1$. Since the underlying probability space $(\Omega, \mathcal{F}, \mathbb{P})$ was assumed to be rich enough to support a random variable with continuous distribution, we may pick a measurable decomposition $\Omega_{1} \cup \Omega_{2} \cup \Omega_{3}$ of $\Omega$ such that $\mathbb{P}\left[\Omega_{1}\right]=x, \mathbb{P}\left[\Omega_{2}\right]=x^{\prime}-x$ and $\mathbb{P}\left[\Omega_{3}\right]=1-x^{\prime}$. We now define random variables $B_{x}:=\mathbb{1}_{\Omega_{1}}, B_{x^{\prime}}:=\mathbb{1}_{\Omega_{1} \cup \Omega_{2}}$ and $B_{x^{\prime}-x}:=\mathbb{1}_{\Omega_{2}}$, and note that they are distributed according to the Bernoulli distribution with parameters $x, x^{\prime}$ and $x^{\prime}-x$, respectively. Moreover we clearly have $B_{x^{\prime}}=B_{x}+B_{x^{\prime}-x}$ on $\Omega$. Thus we 
obtain by Remark 2.13(i) as well as the subadditivity and the distribution-invariance of $\rho_{g}$ that

$$
\begin{aligned}
g\left(x^{\prime}\right)-g(x) & =\rho_{g}\left(F_{-B_{x^{\prime}}}\right)-\rho_{g}\left(F_{-B_{x}}\right) \\
& \leq \rho_{g}\left(F_{-B_{x^{\prime}-x}}\right) \\
& \leq \rho\left(F_{-B_{x^{\prime}-x}}\right) \\
& \leq \sup _{y \in(0,1]} \frac{\rho\left(F_{-B_{y}}\right)}{y^{\beta}}\left(x^{\prime}-x\right)^{\beta} .
\end{aligned}
$$

for every $g \in \mathcal{G}_{\rho}$. Therefore condition (15) implies that every $g \in \mathcal{G}_{\rho}$ is Hölder- $\beta$ continuous on $(0,1]$ with Hölder constant $L$. Since every $g$ is also continuous at 0 , we conclude that every $g \in \mathcal{G}_{\rho}$ is Hölder- $\beta$-continuous even on $[0,1]$ with Hölder constant $L$.

\section{A Examples for distribution-invariant coherent risk measures on Orlicz spaces}

Here we intend to illustrate conditions (11), (12) and (15) by means of examples. At the beginning we will see that the cutting condition (11) and condition (12) are already satisfied if the domain $\mathfrak{X}$ of the coherent risk measure $\rho$ is chosen to be an Orlicz space. Thereafter, in Sections A.1 and A.2, we will give examples for risk measures on Orlisz spaces that satisfy also condition (15). We will show in particular that some of these examples are not covered by the results of Section 2.1 (which justifies Section 2.2).

Recall that a continuous Young function is a convex function $\psi: \mathbb{R}_{+} \rightarrow \mathbb{R}_{+}$satisfying $\psi(0)=0$, being continuous at 0 , and converging to $\infty$ as $x \rightarrow \infty$. Every continuous Young function is in particular nondecreasing. For any continuous Young function $\psi$, the set

$$
\mathcal{L}^{\psi}(\Omega, \mathcal{F}, \mathbb{P}):=\left\{Y \in \mathcal{L}^{0}(\Omega, \mathcal{F}, \mathbb{P}): \mathbb{E}[\psi(|Y| / c)]<\infty \text { for some } c>0\right\}
$$

is known as Orlicz space associated with the Young function $\psi$, whereas the subset

$$
\mathcal{M}^{\psi}(\Omega, \mathcal{F}, \mathbb{P}):=\left\{Y \in \mathcal{L}^{0}(\Omega, \mathcal{F}, \mathbb{P}): \mathbb{E}[\psi(|Y| / c)]<\infty \text { for all } c>0\right\}
$$

is sometimes called Orlicz heart associated with the Young function $\psi$ (cf. [6]). For any continuous Young function $\psi$ we have $\mathcal{L}^{\infty}(\Omega, \mathcal{F}, \mathbb{P}) \subset \mathcal{M}^{\psi}(\Omega, \mathcal{F}, \mathbb{P}) \subset \mathcal{L}^{\psi}(\Omega, \mathcal{F}, \mathbb{P}) \subset$ $\mathcal{L}^{1}(\Omega, \mathcal{F}, \mathbb{P})$. For the particular continuous Young function $\psi(x)=x^{p}$ with $p \in[1, \infty)$, the spaces $\mathcal{M}^{\psi}(\Omega, \mathcal{F}, \mathbb{P})$ and $\mathcal{L}^{\psi}(\Omega, \mathcal{F}, \mathbb{P})$ coincide with $\mathcal{L}^{p}(\Omega, \mathcal{F}, \mathbb{P})$. For details the reader is kindly referred to [30] or [10, Section 2].

Lemma A.1 If $\mathfrak{X}$ is one of the spaces $\mathcal{M}^{\psi}(\Omega, \mathcal{F}, \mathbb{P})$ or $\mathcal{L}^{\psi}(\Omega, \mathcal{F}, \mathbb{P})$ for some continuous Young function $\psi$, then every coherent risk measure $\rho: \mathfrak{X} \rightarrow \mathbb{R}$ satisfies the cutting condition (11) as well as condition (12). 
Proof The sets $L^{\psi}(\Omega, \mathcal{F}, \mathbb{P})$ and $M^{\psi}(\Omega, \mathcal{F}, \mathbb{P})$ of all equivalence classes of $\mathbb{P}$-almost everywhere equal elements of $\mathcal{L}^{\psi}(\Omega, \mathcal{F}, \mathbb{P})$ and $\mathcal{M}^{\psi}(\Omega, \mathcal{F}, \mathbb{P})$ (respectively) are known to be vector spaces. The (Luxemburg) norm,

$$
\|Y\|_{\psi}:=\inf \{c>0: \mathbb{E}[\psi(|Y| / c)] \leq 1\}, \quad Y \in L^{\psi}(\Omega, \mathcal{F}, \mathbb{P})
$$

makes $L^{\psi}(\Omega, \mathcal{F}, \mathbb{P})$ a Banach space with $M^{\psi}(\Omega, \mathcal{F}, \mathbb{P})$ being norm-closed. (Notice that for $\psi(x)=x^{p}$, with $p \in[1, \infty)$, the Luxemburg norm coincides with the classical $L^{p}$. norm $\left.\|\cdot\|_{p} \cdot\right)$ As a consequence $\left(L^{\psi}(\Omega, \mathcal{F}, \mathbb{P}),\|\cdot\|_{\psi}\right)$ and $\left(M^{\psi}(\Omega, \mathcal{F}, \mathbb{P}),\|\cdot\|_{\psi}\right)$ are Banach lattices (with respect to the $\mathbb{P}$-almost sure ordering $\succeq$ ), i.e. Banach spaces such that $\|Y\|_{\psi} \geq\|X\|_{\psi}$ whenever $Y \succeq X$. Therefore every real-valued coherent risk measure $\rho$ on $\mathcal{L}^{\psi}(\Omega, \mathcal{F}, \mathbb{P})$ or $\mathcal{M}^{\psi}(\Omega, \mathcal{F}, \mathbb{P})$ is continuous w.r.t. the seminorm on $\mathcal{L}^{\psi}(\Omega, \mathcal{F}, \mathbb{P})$ associated with $\|\cdot\|_{\psi}$ (cf. [33, Proposition 3.1]).

Below we will show that

$$
\lim _{r \rightarrow \infty}\left\|Y_{r}\right\|_{\psi}=0
$$

for every decreasing sequence $\left(Y_{r}\right)_{r \geq 0} \subset L^{\psi}(\Omega, \mathcal{F}, \mathbb{P})\left(\right.$ resp. $\left.\subset M^{\psi}(\Omega, \mathcal{F}, \mathbb{P})\right)$ converging $\mathbb{P}$-almost surely to 0 as $r \rightarrow \infty$. On the one hand, (20) implies $\lim _{r \rightarrow \infty} \| \lambda(X-$ $r)^{+} \|_{\psi}=0$ for every $X \geq 0$ in $L^{\psi}(\Omega, \mathcal{F}, \mathbb{P})\left(\right.$ resp. in $\left.M^{\psi}(\Omega, \mathcal{F}, \mathbb{P})\right)$ and $\lambda>0$. Along with the seminorm-continuity of the risk measure $\rho$ established above, this would imply that the cutting condition (11) holds. On the other hand, (20) also implies $\lim _{x \rightarrow 0+}\left\|F_{B_{x}}^{\leftarrow}(U)\right\|_{\psi}=0$, where $F_{B_{x}}^{\leftarrow}$ denotes the left-continuous inverse of the df $F_{B_{x}}$ of the Bernoulli distribution with parameter $x$, and $U$ is a random variable on $(\Omega, \mathcal{F}, \mathbb{P})$ which is uniformly distributed on $(0,1)$. Along with the seminorm-continuity of the risk measure $\rho$ established above, this would imply that condition (12) holds.

It remains to show (20). For any decreasing sequence $\left(Y_{r}\right)_{r \geq 0} \subset L^{\psi}(\Omega, \mathcal{F}, \mathbb{P}$ ) (resp. $\left.\subset M^{\psi}(\Omega, \mathcal{F}, \mathbb{P})\right)$ converging $\mathbb{P}$-almost surely to 0 , the sequence $\left(\left\|Y_{r}\right\|_{\psi}\right)_{r \geq 0}$ is decreasing as well. Moreover, for any $0<c \leq \inf _{r}\left\|Y_{r}\right\|_{\psi}$ the decreasing sequence $\left(\psi\left(\left|Y_{r}\right| / c\right)\right)_{r \geq 0}$ converges $\mathbb{P}$-almost surely to 0 due to the continuity of $\psi$. By dominated convergence we deduce $\lim _{r \rightarrow \infty} \mathbb{E}\left[\psi\left(\left|Y_{r}\right| / c\right)\right]=0$, which in turn implies $\inf _{r}\left\|Y_{r}\right\|_{\psi}=0$. This yields in particular (20).

In the following Sections A.1 and A.2, we will present some customary distributioninvariant coherent risk measures on Orlisz spaces that satisfy also condition (15) (apart from conditions (11) and (12)).

\section{A.1 Haezendonck risk measures}

Let $\psi$ be a strictly increasing continuous Young function satisfying $\psi(1)=1$, and let $\mathfrak{X}$ be the Orliz heart $\mathcal{M}^{\psi}(\Omega, \mathcal{F}, \mathbb{P})$ associated with $\psi$. Moreover fix $\alpha \in(0,1)$. It was shown in [14] that for every $X \in \mathcal{M}^{\psi}(\Omega, \mathcal{F}, \mathbb{P})$ and every $x \in \mathbb{R}$ with $\mathbb{P}[X>x]>0$ there exists a unique real number $\pi_{\alpha}^{\psi}(X, x)>x$ such that

$$
\mathbb{E}\left[\psi\left(\frac{(X-x)^{+}}{\pi_{\alpha}^{\psi}(X, x)-x}\right)\right]=1-\alpha .
$$


Therefore we may define a functional $\rho_{\alpha}^{H, \psi}$ on the set $\mathbb{F}^{\psi}$ of all df $F_{X}$ of random variables $X \in \mathcal{L}^{\psi}(\Omega, \mathcal{F}, \mathbb{P})$ by

$$
\rho_{\alpha}^{H, \psi}\left(F_{X}\right):=\inf \left\{\pi_{\alpha}^{\psi}(-X, x): x \in \mathbb{R} \text { with } \mathbb{P}[-X>x]>0\right\}
$$

The functional $\rho_{\alpha}^{H, \psi}$ provides a distribution-invariant positively homogeneous risk measure (cf. [14, Theorem 3.2]) known as Haezendonck risk measure w.r.t. $\psi$ and level $\alpha$. The restriction of $\rho_{\alpha}^{H, \psi}$ to the set of all df of $\mathbb{P}$-essentially bounded random variables has been established to be subadditive in Proposition 12 of [3]. The proof given there can be transferred verbatim to the functional on $\mathcal{L}^{\psi}(\Omega, \mathcal{F}, \mathbb{P})$ itself. So, in fact, $\rho_{\alpha}^{H, \psi}$ provides a distribution-invariant coherent risk measure. According to Lemma A.1 it also satisfies the cutting condition (11) and condition (12). By the way, there is an intimate relationship with Orlicz premiums as introduced in [15]. Indeed: The number $\pi_{\alpha}^{\psi}(X, x)-x$ can be seen as the Orlicz premium of $(X-x)^{+}$w.r.t. $\psi$.

The following lemma shows that under some additional assumptions on $\psi$ the Haezendonck risk measure $\rho_{\alpha}^{H, \psi}$ also satisfies condition (15), so that it meets the requirements discussed subsequent to $(10)$.

Lemma A.2 If there are some $x_{0} \in(0,1-\alpha)$ and $\beta \in(0,1]$ such that

$$
x \psi\left(x^{-\beta}\right) \leq 1-\alpha \quad \forall x \in\left(0, x_{0}\right]
$$

then the Haezendonck risk measure $\rho_{\alpha}^{H, \psi}$ satisfies condition (15). For instance, condition (21) is satisfied for $\psi(x)=x^{p}$ with $p \in[1, \infty)$.

Proof We have for $x \in(0,1)$

$$
\rho_{\alpha}^{H, \psi}\left(F_{-B_{x}}\right) \leq 1 \wedge \pi_{\alpha}^{\psi}\left(B_{x}, x\right)=1 \wedge\left(x+\frac{1-x}{\left.\psi^{-1}((1-\alpha) / x)\right)}\right) .
$$

where $\psi^{-1}$ denotes the inverse of $\psi$ (recall that we assumed the Young function $\psi$ to be strictly increasing). Under assumption (21) we can easily deduce with the help of (14) that

$$
\sup _{x \in(0,1]} \frac{\widetilde{g}_{\rho_{\alpha}^{H, \psi}}(x)}{x^{\beta}}=\sup _{x \in(0,1]} \frac{\rho_{\alpha}^{H, \psi}\left(F_{-B_{x}}\right)}{x^{\beta}} \leq \max \left\{2 ; x_{0}^{-\beta}\right\} .
$$

That is, (15) holds. In the case $\psi(x)=x^{p}$, with $p \in[1, \infty)$, condition (21) is easily seen to hold for $\beta:=(2 p)^{-1}$ and $x_{0}:=(1-\alpha)^{2}$. This verifies the second statement of the lemma.

Now, choose $\psi(x)=x^{p}$ for any $p \in[1, \infty)$. For some choices of $p$ the corrsesponding Haezendonck risk measure degenerates to a distortion risk measure, so that also the results of Section 2.1 could be applied. For instance, for $p=1$ the Haezendonck risk measure $\rho_{\alpha}^{H, \psi}$ is just the Avarage Value-at-Risk at level $1-\alpha$ (see [13, Lemma 4.46] and [3, Section 3.1]). For other choices of $p$, the coherent risk measure $\rho_{\alpha}^{H, \psi}$ does not degenerate to a distortion risk measure (cf. Example A.3). This justifies the investigations of Section 2.2. 
Example A.3 For $\psi(x)=x^{2}$ and $\alpha \in\left(\frac{1}{3}, \frac{3}{8}\right)$ the Haezendonck risk measure $\rho_{\alpha}^{H, \psi}$ is not a distortion risk measure.

Proof According to Remark 2.13(ii) it suffices to show that $\rho:=\rho_{\alpha}^{H, \psi}$ does not coincide with the distortion risk measure $\rho_{\widetilde{g}_{\rho}}$, where $\widetilde{g}_{\rho}$ denotes the distortion function associated with $\rho$ (cf. (13)). We will show that $\rho(-Y) \neq \rho_{\widetilde{g}_{\rho}}(-Y)$ for every random variable $Y$ which is uniformly distributed on $\{0 ; 1 ; 2\}$. On the one hand, for the Haezendonck risk measure we obtain

$$
\rho(-Y) \leq \pi_{\alpha}^{\psi}(Y, 1 / 3)=1 / 3+\left(\frac{\mathbb{E}\left[\left((Y-1 / 3)^{+}\right)^{2}\right]}{(1-\alpha)}\right)^{1 / 2}<5 / 3 .
$$

On the other hand, for the associated distortion function $\widetilde{g}_{\rho}$ we obtain by straightforward calculations

$$
\widetilde{g}_{\rho}(x)=\left\{\begin{array}{cl}
x+\left(\frac{\alpha}{1-\alpha} x(1-x)\right)^{1 / 2} & : x<1-\alpha \\
1 & : x \geq 1-\alpha
\end{array}\right.
$$

and thus

$$
\rho_{\widetilde{g}_{\rho}}(-Y)=\widetilde{g}_{\rho}(1 / 3)+\widetilde{g}_{\rho}(2 / 3)=\frac{2}{3}\left(\frac{2 \alpha}{1-\alpha}\right)^{1 / 2}+1 \geq \frac{5}{3}
$$

Therefore we indeed have $\rho(-Y) \neq \rho_{\widetilde{g}_{\rho}}(-Y)$.

\section{A.2 Risk measures based on one-sided moments}

Let $\mathfrak{X}$ be $\mathcal{L}^{p}(\Omega, \mathcal{F}, \mathbb{P})$ for some $p \in[1, \infty)$ and let $a \in[0,1]$. Then

$$
\left.\rho_{p, a}\left(F_{X}\right):=-\mathbb{E}[X]+a\left(\mathbb{E}\left[(X-\mathbb{E}[X])^{-}\right)^{p}\right]\right)^{1 / p}
$$

defines a distribution-invariant coherent risk measure on the set $\mathbb{F}^{p}$ of all df $F_{X}$ of random variables $X \in \mathcal{L}^{p}(\Omega, \mathcal{F}, \mathbb{P})$ (cf. [12, Lemma 4.1]). The risk measure $\rho_{p, a}$ has been introduced in [12] as a building block for the problem of risk allocation (actually it was studied earlier in $[8]$ on $\mathcal{L}^{\infty}(\Omega, \mathcal{F}, \mathbb{P})$ in a different context). In view of Lemma A.1 it also satisfies the cutting condition (11) and condition (12). Moreover, the following lemma tells us that it also fulfills condition (15), so that it meets the requirements discussed subsequent to (10).

Lemma A.4 The risk measure $\rho_{p, a}$ satisfies condition (15) for every $\beta \in(0,1 / p)$.

Proof It can be easily verified that the associated distortion function $\widetilde{g}_{\rho_{p, a}}$ of $\rho_{p, a}$ satisfies $\widetilde{g}_{\rho_{p, a}}(x)=x+a(1-x) x^{1 / p}$, which immediately yields the statement of the lemma.

In the case $a=0$ the risk measure $\rho_{p, a}$ degenerates to the negative mean which is a distortion risk measure with the identity on $[0,1]$ as distortion function. A similar statement does not hold for $a>0$, as will be shown in the following lemma. 
Lemma A.5 If $a>0$, then the risk measure $\rho_{p, a}$ is not a distortion risk measure.

Proof According to Remark 2.13(ii) it suffices to show that $\rho:=\rho_{p, a}$ does not coincide with the distortion risk measure $\rho_{\widetilde{g}_{\rho}}$, where $\widetilde{g}_{\rho}$ denotes the distortion function associated with $\rho$ (cf. (13)). We will show that $\rho(-Y) \neq \rho_{\widetilde{g}_{\rho}}(-Y)$ for every random variable $Y$ which is uniformly distributed on $\{0 ; 1 ; 2\}$. One the one hand, we have $\rho_{p, a}(-Y)=1+a(1 / 3)^{1 / p}$. On the other hand, we easily obtain $\widetilde{g}_{\rho_{p, a}}(x)=q+a(1-$ $x) x^{1 / p}$, and so

$$
\rho_{\widetilde{g}_{\rho}}(-Y)=\widetilde{g}_{\rho}(1 / 3)+\widetilde{g}_{\rho}(2 / 3)=1+a(1 / 3)^{1 / p}\left(2+2^{\frac{1}{p}}\right) / 3 .
$$

That is, $\rho(-Y) \neq \rho_{\widetilde{g}_{\rho}}(-Y)$.

\section{References}

[1] Artzner, P., Delbaen, F., Eber, J.-M. and Heath, D. (1997) Thinking coherently. Risk, 10(11), 68-71.

[2] Artzner, P., Delbaen, F., Eber, J.-M. and Heath, D. (1999) Coherent measures of risk. Mathematical Finance, 9, 203-228.

[3] Bellini, F. and Rosazza Gianin, E. (2008) On Haezendonck risk measures. Journal of Banking and Finance, 32(6), 986-994.

[4] Bradley, R.C. (2005) Basic properties of strong mixing conditions. A survey and some open problems. Probability Surveys, 2, 107-144.

[5] Carrasco, M. and Chen, X. (2002) Mixing and moment properties of various GARCH and stochastic volatility models. Econometric Theory, 18, 17-39.

[6] Cheridito, P. and Li, T. (2009) Risk mesures on Orlicz hearts. Mathematical Finance, 19(2), 189-214.

[7] Denneberg, D. (1994) Non-additive measure and integral. Kluwer, Dordrecht.

[8] Delbaen, F. (2002) Coherent measures of risk on general probability spaces, in: Sandmann, K. and Schönbucher, P.J. (Eds.) Advances in Finance and Stochastics. Springer-Verlag, Berlin, 1-37.

[9] Doukhan, P. (1994). Mixing: Properties and Examples. Lecture Notes in Statistics, Springer-Verlag, New York.

[10] Edgar, G.A. and Sucheston, L. (1992) Stopping times and directed processes. Cambridge University Press, Cambridge.

[11] Embrechts, P., Klüppelberg, C. and Mikosch, T. (1997) Modelling extremal events: For finance and insurance. Springer-Verlag, New York. 
[12] Fischer, T. (2003) Risk allocation by coherent risk measures based on one-side moments. Insurance: Mathematics and Economics, 32(1), 135-146.

[13] Föllmer, H. and Schied, A. (2004) Stochastic finance. An introduction in discrete time. de Gruyter, Berlin.

[14] Goovaerts, M.J., Kaas, R., Dhaene, J. and Tang, Q. (2004) Some new classes of consistent risk measures. Insurance: Mathematics and Economics, 34(4), 505-516.

[15] Haezendonck, J. and Goovaerts, M.J. (1982) A new premium calculation principle based on Orlicz norms. Insurance: Mathematics and Economics 1, 41-51.

[16] Hardy, M.R. and Wirch, J. (2001) Distortion risk measures: coherence and stochastic dominance. Working Paper, avaible in www.gloriamundi.com.

[17] Hipp, C. (1979). Convergence rates in the central limit theorem for stationary mixing sequences of random variables. Journal of Multivariate Analysis, 9, 560578.

[18] Ibragimov, I.A. (1962) Some limit theorems for stationary sequences. Theory of Probability and its Applications, 7, 349-382.

[19] Jones, B.L. and Zitikis, R. (2004) Empirical estimation of risk measures and related quantities. The North American Actuarial Journal, 8(3), 114-118.

[20] Jones, B.L. and Zitikis, R. (2007) Risk measures, distortion parameters, and their empirical estimation. Insurance: Mathematics and Economics, 41(2), 279-297.

[21] Jouini, E., Schachermayer, W. and Touzi, N. (2006) Law invariant risk measures have the Fatou property, Advances in Mathematical Economics 9, 49-71.

[22] Krätschmer, V. (2007) On $\sigma$-additive robust representation of convex risk measures for unbounded financial positions in the presence of uncertainty about the market model, SFB 649 discussion paper 200\%-010, downloadable at http://sfb649.wiwi.hu-berlin.de.

[23] Kusuoka, S. (2001) On law invariant risk measures. Advances in Mathematical Economics, 3, 505-513.

[24] Lindner, A.M. (2008) Stationarity, mixing, distributional properties and moments of $\operatorname{GARCH}(p, q)$ processes. In: T.G. Andersen, R.A. Davis, J.-P. Kreiß, T. Mikosch (Eds.), Handbook of financial time series, Springer-Verlag, Berlin, 41-69.

[25] Michel, R. (1976) Nonuniform central limit bounds with applications to probabilities of deviations. Annals of Probability, 4, 102-106.

[26] Peligrad, M. (1985) Convergence rates of the strong law for stationary mixing sequences. Zeitschrift für Wahrscheinlichkeitstheorie und verwandte Gebiete, 70, 307-314. 
[27] Peligrad, M. and Shao, Q.M. (1993) Self-normalized central limit theorem for sums of weakly dependent random variables. Journal of Theoretical Probability, $7(2), 309-338$.

[28] Pflug, G. and Römisch, W. (2007) Modelling, managing and measuring risk. World Scientific, Singapore.

[29] Philipp, W. and Stout, W.F. (1975) Almost sure invariance principles for partial sums of weakly dependent random variables. Memoirs of the American Mathematical Society, 2(161), Providence.

[30] Rao, M.M. and Ren, Z.D. (1991) Theory of Orlicz spaces. Marcel Dekker, New York.

[31] Rio, E. (1994) A maximal inequality and dependent Marcinkiewicz-Zygmund strong laws. Annals of Probability, 23, 918-937.

[32] Rosenblatt, M. (1956) A central limit theorem and a mixing condition. Proceedings of the National Academy of Sciences of the USA, 42, 412-413.

[33] Ruszczynski, A. and Shapiro, A. (2006) Optimization of convex risk functions. Mathematics of Operations Research, 31, 433-451.

[34] Schneider, E. (1981) On the speed of convergence in the random central limit theorem for $\varphi$-mixing processes. Zeitschrift für Wahrscheinlichkeitstheorie und verwandte Gebiete, 58, 125-138.

[35] Stout, W.F. (1974) Almost sure convergence. Academic Press, New York.

[36] Wang, S. (1996) Premium calculation by transforming the layer premium density. ASTIN Bulletin, 26, 71-92.

[37] Wang, S. and Dhaene, J. (1998) Comontonicity, correlation order and premium principles. Insurance: Mathematics and Economics, 22, 235-242.

[38] Zähle, H. (2010) Rates of almost sure convergence of plug-in estimates for distortion risk measures. Metrika, forthcoming. 

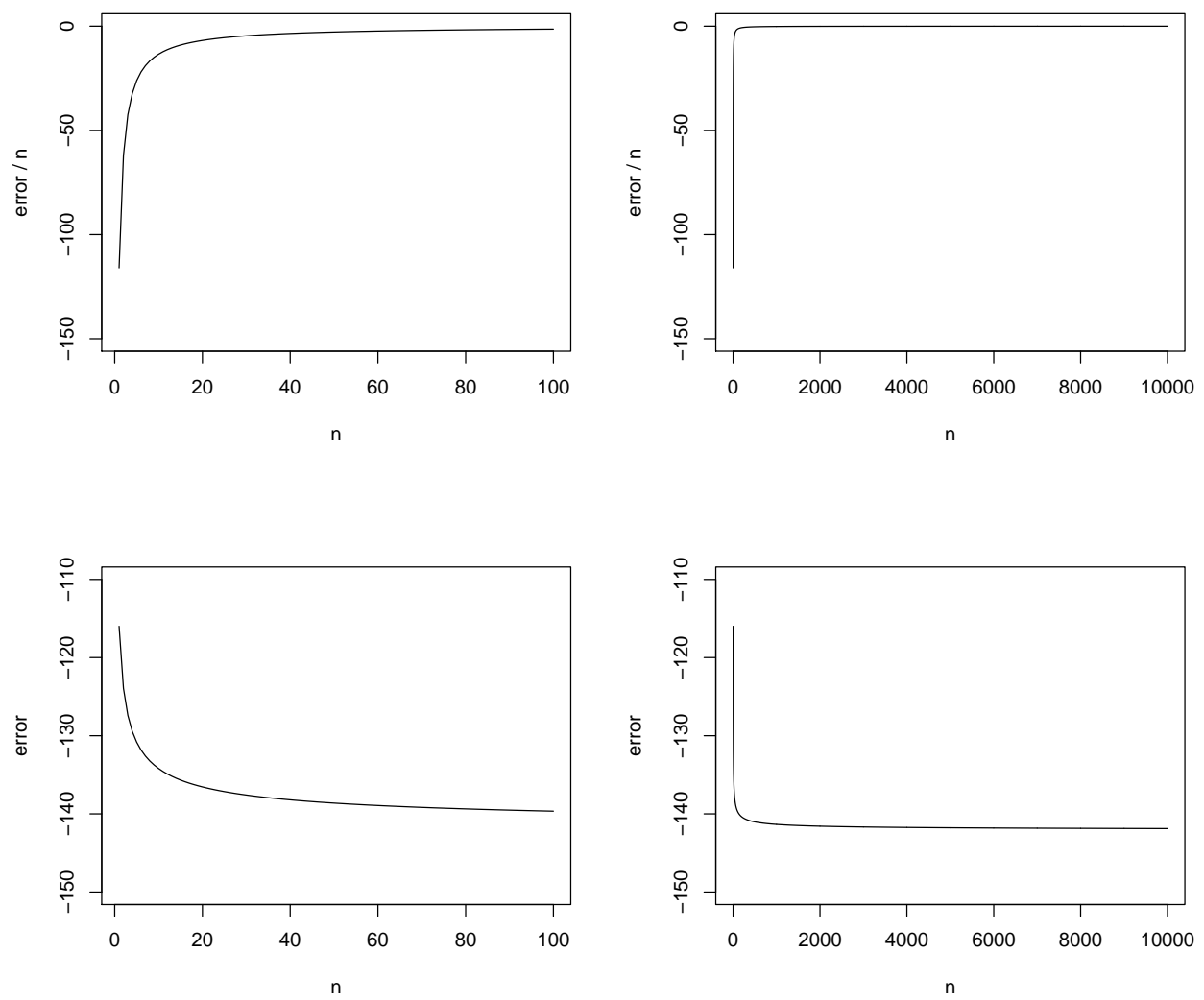

Figure 1: In each column the curves $n \mapsto\left(\rho\left(\Phi_{n}\right)-\rho\left(F_{n}\right)\right) / n$ and $n \mapsto\left(\rho\left(\Phi_{n}\right)-\rho\left(F_{n}\right)\right)$ are displayed, where $\rho$ is the Value-at-Risk at level $\alpha=0.05$, and $F_{1}$ is the Gamma df on $\mathbb{R}_{-}$with parameters $a=4$ and $b=0.004$. 

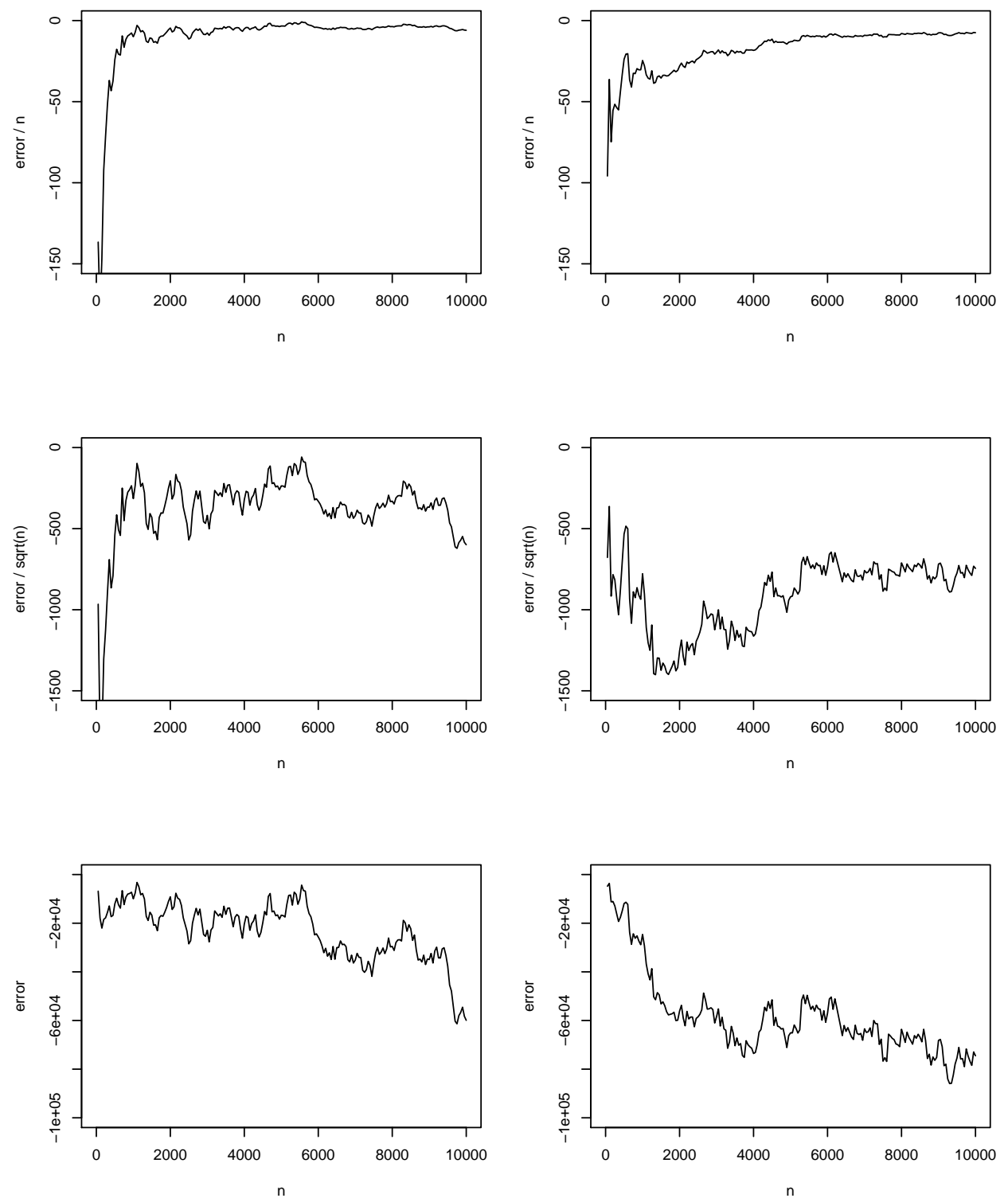

Figure 2: Sample paths of $n \mapsto\left(\rho\left(\hat{\Phi}_{n}\right)-\rho\left(F_{n}\right)\right) / n, n \mapsto\left(\rho\left(\hat{\Phi}_{n}\right)-\rho\left(F_{n}\right)\right) / \sqrt{n}$ and $n \mapsto\left(\rho\left(\hat{\Phi}_{n}\right)-\rho\left(F_{n}\right)\right)$, where $\rho$ is the Value-at-Risk at level $\alpha=0.05, F_{1}$

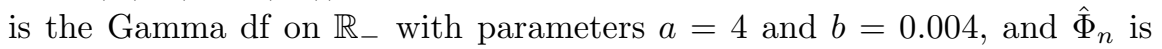
based on $n$ simulated i.i.d. $F_{1}$ random variables (the two columns mirror two different Monte Carlo simulations). 


\section{SFB 649 Discussion Paper Series 2010}

For a complete list of Discussion Papers published by the SFB 649, please visit http://sfb649. wiwi.hu-berlin. de.

001 "Volatility Investing with Variance Swaps" by Wolfgang Karl Härdle and Elena Silyakova, January 2010.

002 "Partial Linear Quantile Regression and Bootstrap Confidence Bands" by Wolfgang Karl Härdle, Ya'acov Ritov and Song Song, January 2010.

003 "Uniform confidence bands for pricing kernels" by Wolfgang Karl Härdle, Yarema Okhrin and Weining Wang, January 2010.

004 "Bayesian Inference in a Stochastic Volatility Nelson-Siegel Model" by Nikolaus Hautsch and Fuyu Yang, January 2010.

005 "The Impact of Macroeconomic News on Quote Adjustments, Noise, and Informational Volatility" by Nikolaus Hautsch, Dieter Hess and David Veredas, January 2010.

006 "Bayesian Estimation and Model Selection in the Generalised Stochastic Unit Root Model" by Fuyu Yang and Roberto Leon-Gonzalez, January 2010.

007 "Two-sided Certification: The market for Rating Agencies" by Erik R. Fasten and Dirk Hofmann, January 2010.

008 "Characterising Equilibrium Selection in Global Games with Strategic Complementarities" by Christian Basteck, Tijmen R. Daniels and Frank Heinemann, January 2010.

009 "Predicting extreme VaR: Nonparametric quantile regression with refinements from extreme value theory" by Julia Schaumburg, February 2010.

010 "On Securitization, Market Completion and Equilibrium Risk Transfer" by Ulrich Horst, Traian A. Pirvu and Gonçalo Dos Reis, February 2010.

011 "Illiquidity and Derivative Valuation" by Ulrich Horst and Felix Naujokat, February 2010.

012 "Dynamic Systems of Social Interactions" by Ulrich Horst, February 2010.

013 "The dynamics of hourly electricity prices" by Wolfgang Karl Härdle and Stefan Trück, February 2010.

014 "Crisis? What Crisis? Currency vs. Banking in the Financial Crisis of 1931" by Albrecht Ritschl and Samad Sarferaz, February 2010.

015 "Estimation of the characteristics of a Lévy process observed at arbitrary frequency" by Johanna Kappusl and Markus Reiß, February 2010.

016 "Honey, I'll Be Working Late Tonight. The Effect of Individual Work Routines on Leisure Time Synchronization of Couples" by Juliane Scheffel, February 2010.

017 "The Impact of ICT Investments on the Relative Demand for HighMedium-, and Low-Skilled Workers: Industry versus Country Analysis" by Dorothee Schneider, February 2010.

018 "Time varying Hierarchical Archimedean Copulae" by Wolfgang Karl Härdle, Ostap Okhrin and Yarema Okhrin, February 2010.

019 "Monetary Transmission Right from the Start: The (Dis)Connection Between the Money Market and the ECB's Main Refinancing Rates" by Puriya Abbassi and Dieter Nautz, March 2010.

020 "Aggregate Hazard Function in Price-Setting: A Bayesian Analysis Using Macro Data" by Fang Yao, March 2010.

021 "Nonparametric Estimation of Risk-Neutral Densities" by Maria Grith, Wolfgang Karl Härdle and Melanie Schienle, March 2010. 


\section{SFB 649 Discussion Paper Series 2010}

For a complete list of Discussion Papers published by the SFB 649, please visit http://sfb649. wiwi. hu-berlin.de.

022 "Fitting high-dimensional Copulae to Data" by Ostap Okhrin, April 2010.

023 "The (In)stability of Money Demand in the Euro Area: Lessons from a Cross-Country Analysis" by Dieter Nautz and Ulrike Rondorf, April 2010.

024 "The optimal industry structure in a vertically related market" by Raffaele Fiocco, April 2010.

025 "Herding of Institutional Traders" by Stephanie Kremer, April 2010.

026 "Non-Gaussian Component Analysis: New Ideas, New Proofs, New Applications" by Vladimir Panov, May 2010.

027 "Liquidity and Capital Requirements and the Probability of Bank Failure" by Philipp Johann König, May 2010.

028 "Social Relationships and Trust" by Christine Binzel and Dietmar Fehr, May 2010.

029 "Adaptive Interest Rate Modelling" by Mengmeng Guo and Wolfgang Karl Härdle, May 2010.

030 "Can the New Keynesian Phillips Curve Explain Inflation Gap Persistence?" by Fang Yao, J une 2010.

031 "Modeling Asset Prices" by James E. Gentle and Wolfgang Karl Härdle, June 2010.

032 "Learning Machines Supporting Bankruptcy Prediction" by Wolfgang Karl Härdle, Rouslan Moro and Linda Hoffmann, J une 2010.

033 "Sensitivity of risk measures with respect to the normal approximation of total claim distributions" by Volker Krätschmer and Henryk Zähle, June 2010. 